\title{
Laboreal
}

Volume $14 \mathrm{~N}^{\circ} 2$ | 2018

Digitalização e evolução do trabalho real

\section{Profesiones de servicio y digitalización: implicaciones y sugerencias para la concepción de procesos de formación}

Profissões de serviços e digitalização: implicações e sugestões para a conceção de programas de formação

Métiers de services et numérisation: implications et suggestions pour la conception de dispositifs de formation

Professions from the services sector and digitalization: implications and guidelines for the design of training programs

Deli Salini, Juana Sarmiento Jaramillo, Annie Goudeaux y Germain Poizat

\section{OpenEdition}

Edición electrónica

URL: http://journals.openedition.org/laboreal/584

DOI: $10.4000 /$ laboreal.584

ISSN: 1646-5237

Editor

Universidade do Porto

Referencia electrónica

Deli Salini, Juana Sarmiento Jaramillo, Annie Goudeaux y Germain Poizat, « Profesiones de servicio y digitalización: implicaciones y sugerencias para la concepción de procesos de formación », Laboreal [En línea], Volume 14 No2 | 2018, Publicado el 01 diciembre 2018, consultado el 10 diciembre 2020. URL : http://journals.openedition.org/laboreal/584 ; DOI : https://doi.org/10.4000/laboreal.584

Este documento fue generado automáticamente el 10 diciembre 2020.

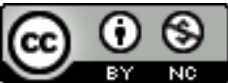

Laboreal está licenciado com uma Licença Creative Commons - Atribuição-NãoComercial 4.0 Internacional. 


\section{Profesiones de servicio y digitalización: implicaciones y sugerencias para la concepción de procesos de formación}

Profissões de serviços e digitalização: implicações e sugestões para a conceção de programas de formação

Métiers de services et numérisation: implications et suggestions pour la conception de dispositifs de formation

Professions from the services sector and digitalization: implications and guidelines for the design of training programs

Deli Salini, Juana Sarmiento Jaramillo, Annie Goudeaux y Germain Poizat

\section{NOTA DEL EDITOR}

http://dx.doi.org/10.15667/laborealxiv218ds

Manuscrito recebido em: julho/2018

Aceite após peritagem: outubro/2018

\section{Introducción}

1 El fenómeno de la digitalización tiene consecuencias importantes y complejas para la sociedad y genera, entre otros, el surgimiento de una gobernabilidad algorítmica, de una sociedad de híper control, y la potencial desaparición del trabajo (e.g. Benghozi, 2011; Benghozi, Gille, \& Vallée, 2009; Poizat \& Durand, 2017; Stiegler, 2015a). Este impacto también se manifiesta en las profesiones de servicio. En este campo, la digitalización, entendida como espacio de innovación, está llamada a acompañar y 
dinamizar los esfuerzos de productividad impuestos por las prácticas gerenciales, que buscan entre otros, una reducción en tiempo y costos y una mejora de las relaciones con los usuarios (Djellal \& Gallouj, 2007).

2 Frente a los cuestionamientos relativos a la relación entre el trabajo y la formación, postulamos la tecnología como antropológicamente constitutiva y constituyente de la actividad humana (Steiner, 2010), y la abordamos como un pharmakon (Stiegler, 2015a, 2010/2015b), es decir como cura y veneno al mismo tiempo, potencialmente portadora de emancipación e individuación, pero también de alienación, proletarización y desindividuación.

3 Con el fin de explorar y discutir estas implicaciones, esta contribución se enfoca en la influencia de la digitalización en el sector particular de las 'actividades de servicio', en las que los profesionales interactúan directamente con los usuarios no profesionales con el fin de ofrecerles un servicio que de una u otra manera responde a las necesidades de estos últimos (Falzon \& Lapeyrière, 1998). La relación de servicio es un 'encuentro con una finalidad', inexorablemente guiada por el vínculo con el otro, donde la relación está tecnificada o mediada por la técnica. En este sentido, es el resultado de una historia co-construida entre el operador y el usuario. Por lo demás, la relación va mucho más allá de esta interacción particular, en la medida en que se basa en una actividad - visible o invisible - que es individual y colectiva a la vez (Caroly \& WeillFassina, 2007; Motté \& Haradji, 2010) y que se inscribe en un entorno técnicoorganizacional (Falzon et al., 1998; Valléry \& Leduc, 2005). En el campo de la prestación de servicios, la difusión de la tecnología digital corresponde usualmente a la implementación progresiva de herramientas digitales que buscan facilitar la gestión y transmisión de la información (Djellal et al., 2007).

4 Este artículo presenta dos investigaciones empíricas preliminares realizadas en Suiza en el marco de dos profesiones de servicio, y aborda particularmente los aspectos relacionados con la integración de Objetos Técnicos Digitales (OTD) en el proceso de documentación de la información de los usuarios y las propuestas iniciales de formación dirigidas a los profesionales interesados. La primera investigación (Investigación A) se refiere a las prácticas de enfermería (Salini, 2018) y la segunda (Investigación/intervención B) a aquellas de los agentes judiciales de embargo [1] (Durand, Goudeaux, Poizat \& Sarmiento Jaramillo, aceptado). El paralelo de estos dos estudios es pertinente puesto que ambos: a) conciernen profesiones de servicio que implican la presencia de OTD para la gestión y transmisión de datos de los usuarios; b) se basan en el mismo marco teórico y metodológico de antropo-tecnología educativa; c) buscaron identificar aspectos típicos de la actividad de los actores implicados; d) buscaron generar pistas útiles para la concepción de propuestas de formación. Dado su carácter exploratorio, estas investigaciones no tuvieron en cuenta datos relativos a la actividad de los usuarios, y por lo tanto no pueden dar testimonio de la dimensión coconstruida de la relación de servicio.

5 La Investigación A describe en qué medida los OTD ligados a los procesos de transmisión de las Informaciones Clínicas de los Pacientes (ICP) a) materializan un doble monitoreo del estado de salud del paciente y de la actividad de enfermería; b) constriñen el trabajo de enfermería a través de la instauración de una red socio-técnica ampliada, integrando otros soportes de gestión y transmisión de la información; c) modifican profundamente los modos de transmisión de las ICP. Estos elementos, que presentan a la vez dimensiones críticas y aspectos de desarrollo, invitan a replantear de 
cero la correlación entre las dimensiones técnica y relacional en el trabajo de cuidados sanitarios y cuestionan las relaciones intergeneracionales y la conexión de los profesionales con el futuro. En este sentido, las propuestas de formación que surgen conciernen diferentes modalidades de promoción de una reflexividad aumentada acerca de la actividad profesional y el acompañamiento al desarrollo de un enfoque prospectivo (Godet \& Durance, 2011; Poli, 2017).

6 La investigación B se refiere a una intervención piloto realizada en un servicio público en el que la actividad de los profesionales se alteró debido a (aunque no exclusivamente) la implementación de un nuevo progicial, que reemplazó el software que se usaba para la recopilación de datos de los deudores y el procesamiento de su expediente digitalizado. Esta investigación muestra la principal transformación de la actividad de los agentes judiciales de embargo (AJE) y la manera en la que este nuevo progicial a) tiende a aminorar tanto el proceso de constitución de la información como su complejidad; y b) supone el riesgo de reducir la actividad de los AJE a la sola memorización de informaciones fácticas a consignar en un formulario digital y de reducir la complejidad de la indagación que estos llevan a cabo. Debido a las dificultades experimentadas por la organización y al desánimo de los AJE en su trabajo (pérdida de sentido basada entre otras cosas en el corto circuito producido por ese circuito hipomnésico), se realizó una primera intervención de carácter formativo, según una modalidad alejada de las formaciones curriculares estándar. La formación no tenía como objetivo una adaptación del trabajo al progicial, sino más bien una reactivación del proceso de individuación con miras a un desarrollo (Poizat et al., 2017; Poizat \& Goudeaux, 2014).

$7 \quad$ El artículo inicia con la presentación del marco teórico y metodológico que fundamenta las dos investigaciones. A continuación, se presentan para cada una de ellas el contexto, la metodología de recolección de datos, los resultados y sus implicaciones. La discusión final aborda los aspectos que con la presencia de OTD en la actividad laboral se ponen en juego, los riesgos inherentes a las transformaciones asociadas a estas prácticas de digitalización, y presenta nuestra perspectiva con respecto a propuestas de procesos de formación.

\section{Una perspectiva de antropo-tecnología éducativa}

8 Las investigaciones aquí presentadas se inscriben en una perspectiva de antropotecnología educativa (Poizat et al., 2017; Theureau, 2012, 2015), en el marco de investigaciones interdisciplinarias que articulan investigación empírica e investigación tecnológica.

9 La noción de antropo-tecnología fue propuesta por Wisner (1984/2012, 1997), quien se interesó en la transferencia de tecnologías hacía países en vías de desarrollo. Pariente de la ergonomía, Geslin considera que ésta se orienta hacia la resolución de problemas que supone la llegada de una nueva tecnología a un nuevo contexto, diferente a aquel que le dio vida, (2006, p. 149, traducción personal). Esta concepción original, que tiene un alcance programático importante (Geslin, 2005, 2012), es aquí reconsiderada en tanto que antropo-tecnología educativa a la luz de los avances científicos actuales y de las problemáticas de la formación. Nuestra perspectiva, que se inscribe en los desarrollos recientes del programa de investigación 'curso de la acción' (Theureau, 2012, 2015), tiene una doble referencia: a) a la corriente de la ergonomía francesa, que 
subraya la distancia irreductible entre las tareas prescritas y las actividades realizadas, el carácter enigmático de las situaciones de trabajo y el análisis del trabajo como condición previa para la formación (de Montmollin, 1986), y que lleva su objeto de estudio más allá del análisis de la actividad a fin de apuntarle a la actividad en todo contexto (Theureau, 2006), y b) a los estudios en formación de adultos y en aprendizajedesarrollo en el trabajo que subrayan la opacidad y la dimensión tácita de la actividad profesional (Bourgeois \& Durand, 2012 ; Durand \& Filliettaz, 2009).

contexto, nuestro enfoque de antropo-tecnología educativa tiene como fundamento tanto una ontología como una epistemología enactivas de la actividad (Durand, 2009 ; Theureau, 2006, 2015), en el cual consideramos como hipótesis significativas para nuestro propósito: a) con relación al postulado de la enacción, la dimensión cognitiva, significante, autónoma, corporizada y situada de la actividad; b) la dimensión vivencial de la actividad que se acompaña de una experiencia e implica una dinámica de significación; c) la dimensión antropológicamente constitutiva y constituyente de la técnica (incluida la tecnología digital); d) una concepción de los dispositivos de formación como objetos técnicos que le apuntan al desarrollo de la actividad humana.

11 La noción de enacción fue propuesta por Varela con el fin de subrayar la idea de que la cognición se manifiesta como una propiedad emergente de la historia del acoplamiento de un organismo con su entorno (Varela, Thompson \& Rosch, 1991/1992). Esta interacción entre el organismo y el entorno es in-formativa, de suerte que el organismo se configura o se auto-construye en ese acoplamiento mientras configura su entorno, en una relación que es siempre asimétrica, es decir, orientada por su propia perspectiva. Esta concepción niega cualquier separación entre el cuerpo y el espíritu, al acentuar la dimensión encarnada y corporizada del conocimiento y su carácter irreductiblemente situado en el mundo y en el tiempo (Theureau, 2012).

La experiencia se considera a la vez como la vivencia de la actividad y como el flujo interpretativo continuo de la existencia (Theureau, 2006). En tanto vivencia de la actividad, responde a una modalidad particular de conciencia de 'presencia de sí mismo' que es consubstancial al curso de la actividad, y que tiene como origen una vivencia en primera persona. Incluye igualmente una dinámica de significación, que se constituye en la interacción de las tres categorías descritas por Pierce (1994): a) aquella de lo 'Posible', o las significaciones que podrían o no actualizarse en una situación determinada; b) aquella de lo 'Actual' o de lo que es significativo en una situación específica; c) aquella de lo 'Virtual' o de la generalización que emerge de la interacción entre las dos categorías precedentes. La integración de esta perspectiva semiótica funda el marco teórico de la 'semiología de la actividad' (Theureau, 2006).

13 La hipótesis de « la técnica antropológicamente constitutiva' recalca el hecho de que la técnica es inherente a la actividad humana y es vector determinante de humanización individual y colectiva (Steiner, 2010; Stiegler, 2014, 2015a). En este sentido, participa de la génesis de los procesos psíquicos, de la socialidad y de la historicidad (Havelange, 2005). Al transformar la actividad humana, genera nuevas prácticas, media la creación de comunidades y, más ampliamente, transforma las relaciones sociales. La técnica es también constituyente en cuanto es 'un faire advenir, un faire être' (Steiner, 2010, p. 28), y abre a 'un devenir' en la medida en que la individuación humana (individual o colectiva) está íntimamente ligada a la técnica y a la relación que con ella se establece (Simondon, 1989/2008, 2005). 

que deben considerarse como de 'doble filo' (Poizat et al., 2017, p. 20) y manifiestan una ambivalencia digital originaria e irreductible. Así, el carácter virtuoso o no de las prácticas ligadas a la tecnología digital es una cuestión de dosificación y de voluntad. necesidad de identificar las condiciones de valorización / desvalorización del recurso a la técnica, y de explorar las maneras en las que una comunidad integra una nueva tecnología (inclusive en los procesos de innovación local), y cómo esta tecnología puede adaptarse a las comunidades a las que se destina. Todo lo anterior en consonancia con una perspectiva respetuosa de las maneras en las que los individuos actúan en sus contextos ecológicos (Geslin, 2002). desarrollar un programa de investigación tecnológica en el campo de la educación / formación (Durand, 2009).

Esto implica a) concebir la formación como una tecnología y articular la actividad de concepción al análisis detallado y a la modelización de la actividad de los individuos implicados; b) tomar como objeto de concepción la situación de apropiación; y c) considerar la formación como una actividad prospectiva que permita a los profesionales eliminar las trabas propias de los procesos de apropiación - alienación / individuación en los que existe el riesgo de que la tecnología digital impacte negativamente. En este sentido, los procesos de formación no le apuntan a una adaptación 'reactiva' a los OTD, sino más bien constituyen espacios de problematización sobre la manera en que estos objetos interactúan con la actividad de los individuos, con el fin de identificar los procesos de transformación de sus modalidades de participación en el acoplamiento actor-entorno (Poizat et al., 2014).

\section{Metodología}

Basados en metodologías de observación y análisis in situ del trabajo, nuestras investigaciones le apuntan a evidenciar y convertir en analizables las dimensiones implícitas de la actividad humana que fundamentan la concepción de procesos de formación innovadores. Los datos recolectados a partir de elementos contextuales y de la observación de los profesionales que participaron en la investigación (aspectos extrínsecos de la actividad) se articulan con el contenido de la experiencia expresada por los participantes (dimensiones intrínsecas de la actividad), a la que se otorga siempre la prevalencia (Theureau, 2006). En lo que sigue, presentamos el proceso metodológico común a las dos investigaciones.

En primer lugar, es esencial asegurar unas condiciones éticas y metodológicas específicas con el fin de garantizar la fiabilidad de los datos recolectados. La misma requiere un compromiso de parte del investigador de establecer una relación con el otro alejada de la dominación o la 'objetivación'. Se materializa en: a) la elaboración de un contrato explícito de cooperación entre investigadores y actores del terreno; b) la cooperación entre los diferentes actores a lo largo de toda la investigación para definir las condiciones de la misma y el objeto de estudio; c) la presentación de los resultados a los actores del terreno; d) la suspensión de juicios de valor, es decir, la inhibición (al menos momentánea) de una actividad evaluativa de parte tanto del investigador como 
de los actores respecto de las características de la actividad analizada (Durand, 2009 ; Theureau, 2006, 2015).

La dimensión intrínseca de la actividad se estudia a través de la descripción de la actividad desde el punto de vista del actor, que se operacionaliza a partir del contenido de su 'conciencia prerreflexiva' (Sartre, 1943/2004) y de la consideración de lo que al actor le resulta significativo, mostrable, narrable, imitable y comentable. La expresión de esta conciencia prerreflexiva se realiza en el marco de sesiones de autoconfrontación a los huellas de su actividad, es decir, a las notas de terreno del investigador, los extractos de documentos que provengan del contexto y/o las grabaciones de video. En estas sesiones (grabadas en video) los actores son invitados a 'revivir', en presencia del investigador, las situaciones y su actividad pasada, e incitados a narrar, mostrar, describir y comentar los elementos que les son significativos (Theureau, 2006, 2010).

21 El conjunto de elementos extrínsecos e intrínsecos así recolectados, se retoman durante el análisis de datos a través de la elaboración de una síntesis que se lleva a cabo en varias etapas. Inicialmente, se reconstruyen los elementos típicos de la actividad y de la experiencia vivida de cada actor implicado, relativos a los componentes del marco teórico de semiología de la actividad. Estos componentes corresponden aproximadamente a lo que se entiende como intenciones, expectativas, saberes, percepciones, sensaciones y recuerdos, acciones (simbólicas o prácticas) y generalizaciones (Theureau, 2006). Luego se identifican las similitudes y las singularidades entre los subgrupos (varios profesionales y varias situaciones). Finalmente, se procede a la identificación y reconstitución de episodios-tipo conceptualizados como 'ejemplos ejemplares', es decir, como los mejores representantes posibles de la muestra de las actividades observadas (Schütz, 2010; Theureau, 2006), y por extensión, del trabajo en cuestión.

El carácter de tipicidad remite a cinco aspectos identificados en la muestra de actores y situaciones estudiadas: descriptivo (concentración de atributos de la actividad observada), estadístico (frecuencia observada), generativo (propensión a actualizarse de manera privilegiada cuando se reproducen condiciones similares), significativo (los actores expresan un sentimiento de tipicidad cuando se les interroga al respecto), y expansivo (el análisis muestra ajustes, migraciones y diversificaciones de los tipos en el tiempo y según los entornos) (Durand, 2014; Poizat, Durand \& Theureau, 2016).

\section{Investigación a: « práctica de enfermería y dispositivos digitales »}

Esa investigación tenía como propósito acotar problemáticas de investigación e intervención concernientes a la influencia de la difusión de los OTD para la información y la comunicación en la práctica de la enfermería. Lo anterior con el fin de actualizar los contenidos y las modalidades de formación de base y continua de este personal (Salini, 2008). Los principales temas de la investigación se referían a las características de la difusión de los OTD en la práctica cotidiana de las enfermeras y a su percepción de la influencia de estos objetos en su práctica. 


\subsection{Contexto y modalidad de la realización de la investigación}

El trabajo de campo se llevó a cabo en cuatro servicios de cuidados sanitarios de la parte italohablante de Suiza. Se realizaron casi cien horas de observación in situ de quince profesionales (tres días por servicio), complementadas con notas de terreno y entrevistas a veinte enfermeras (estas entrevistas fueron grabadas y transcritas). Las entrevistas siguieron una trama que abordaba elementos de orden general (p. ej., biografía profesional y relación con la tecnología digital en la vida profesional), elementos específicos de la actividad cotidiana (para el caso de las personas observadas en su trabajo a partir de auto-confrontaciones a huellas de la actividad; evocando ciertos elementos observados; $\mathrm{y}$, para todas, la exhortación a citar situaciones específicas vividas relativas al uso de los OTD), y finalmente elementos de orden prospectivo (relativos a la evolución de los cuidados de enfermería luego de la digitalización).

\subsection{Resultados}

Dado que las enfermeras deben « lidiar » con un sistema digital multinivel de gestión de información y comunicación en los servicios de cuidados sanitarios, el análisis de su utilización no puede disociar los dispositivos digitales individuales (PC, tabletas, Laptop) del uso del progicial utilizado para la gestión del proceso de transmisión de Informaciones Clínicas de los Pacientes (ICP). Este progicial difería según los servicios y según su grado de implementación (presencia o no de ciertas extensiones o relación con otras aplicaciones).

En la práctica de enfermería el proceso de transmisión de las ICP es fundamental, pues implica una transferencia de las responsabilidades y obligaciones profesionales a otra persona o a un grupo profesional respecto de todos o una parte de los cuidados de los pacientes (Australian Commission on Safety and Quality in Health Care, 2010). Con respecto a este proceso y a las implicaciones de los OTD utilizados, el análisis permitió identificar tres ejes temáticos principales a) la presencia de un doble monitoreo del estado de salud del paciente y de la actividad de enfermería; b) la presencia de una multiplicidad de soportes a los cuales se integran los OTD; c) la modificación sustancial de las modalidades de transmisión (oral y/o escrita en diferentes soportes) de las ICP. Estas temáticas se relacionan con algunas más generales relativas a la apropiación individual y colectiva de los OTD para las IPC, a las relaciones intergeneracionales, y a la relación que con el futuro establecen los profesionales.

\subsubsection{La concretización de un doble monitoreo}

Los OTD utilizados en la gestión de las IPC a) facilitan y aceleran la transmisión, organización y monitoreo continuo de las informaciones sobre la salud y los cuidados del paciente; $y b$ ) hacen posible el monitoreo continuo de las prestaciones del personal de enfermería. A través de esos OTD el personal de enfermería reúne, analiza o transmite a la vez informaciones relativas a los datos administrativos y clínicos de los pacientes y a los de la actividad de enfermería. Los 'datos paciente' están destinados a asegurar de parte de todas enfermeras un monitoreo regular de la situación de los pacientes, con el fin de garantizar la continuidad de los cuidados y su trazabilidad; los 'datos enfermera' relacionados con los pacientes, permiten detectar e identificar las 
necesidades de personal y los costos de las prestaciones realizadas. El hecho de que estos elementos sean recolectados conjuntamente por una única herramienta parece facilitar la organización, transparencia y transmisión de la información, así como el control de la calidad de los cuidados, de la gestión financiera y del personal.

Sin embargo, el personal de enfermería manifiesta ciertas preocupaciones:

- El trabajo de cuidados sanitarios parece avanzar hacia otorgarle una prioridad a las dimensiones procedurales y la recolección/transmisión de datos por sobre la relación real con los pacientes y la prestación de los servicios de enfermería. Esta vivencia se acompaña por la preocupación que genera una obligación velada de acelerar los tiempos de cuidados o de contacto relacional con los pacientes, a fin de reducir los recursos en términos de personal y costos. Si bien parcialmente desmentido por los líderes, esto constituye un rumor bien difundido.

- Se menciona un riesgo creciente de efecto paradoxal, presente especialmente cuando el trabajo asistencial aumenta (por ejemplo en situaciones críticas para los pacientes o en el caso de insuficiencia puntual de efectivos). Aquí, el aumento de la carga de trabajo se acompaña de una disminución en el detalle con el que se reseñan las IPC en los expedientes: debido a la falta de tiempo, la redacción de estos datos se deja para más tarde (particularmente al final de la jornada, lo que genera a veces horas de trabajo suplementarias), y/o se sintetiza (no todos los detalles se especifican). Así, mientras más aumenta el trabajo, mayor es el riesgo de no documentar integral y exhaustivamente. Como lo indica una enfermera: 'Los tiempos para hacer lo que se debe hacer están prefijados (...) y deben modificarse en caso de aumento. Pero es ahí justamente donde está el problema, pues muchas veces tenemos tantas cosas que hacer y quieres simplemente terminar con el computador personal, que no registras el aumento...'

\subsubsection{Una red ampliada de gestión y transmisión de información}

Si bien una de las finalidades de la introducción de los OTD es la transformación del flujo de documentos en papel a un flujo electrónico, los datos guardados o transmitidos digitalmente coexisten actualmente con una serie de informaciones adicionales plasmadas en una multiplicidad de tipos de soporte. Así, los OTD se articulan con a) expedientes en papel utilizados para prescripciones médicas que requieren firmas; $b$ ) diferentes formatos o cuadros con información adosados a los muros de las dependencias de enfermería (o en las habitaciones de los pacientes) relativos a la organización del trabajo y a las personas de referencia de los diferentes pacientes; y c) toda suerte de formularios (exámenes clínicos, pedidos de medicamentos, dietas, etc.) que deben presentarse. Esta documentación, que se actualiza regularmente, es complementada con algunos documentos en papel de carácter personal generados por las enfermeras, que resumen ciertos elementos clave de las intervenciones a realizar o de los aspectos a vigilar, así como por pequeñas notas de papel producidas a lo largo de toda la jornada.

30 La introducción progresiva de las funciones del progicial en los servicios hace que el nivel de digitalización de la información varíe según el tiempo y el lugar. La adaptación de la documentación digital a las especificidades y complejidades de los servicios es la causa de esta introducción gradual.

31 La multiplicidad de medios técnicos de comunicación mencionada, así como la actualización continua del sistema de gestión de las ICP, suponen de parte del personal una atención constante al registro de información en los diferentes medios de soporte, 
a la actualización de las tipologías de soportes (por ej., reedición de los formularios) y a los cambios regulares de procedimientos de gestión de datos. En paralelo, se requiere una actualización continua de sus conocimientos relativos a las técnicas de cuidado y de adaptación a los diferentes niveles mencionados, lo cual incrementa la carga cotidiana de trabajo.

Frente a este proceso de actualización continua, algunas experimentan sentimientos de inseguridad y miedo de no estar a la altura o de cometer errores: 'a veces olvido anotarlo todo, o la forma de registrar algunos datos' dice una enfermera; otra precisa 'hay demasiadas cosas, demasiados programas, demasiados objetos'.

\subsubsection{Modificaciones de las modalidades de transmisión de las Informaciones Clínicas de los Pacientes}

Las reuniones de relevo propias de los momentos de cambio de equipo caracterizan en parte el trabajo de enfermería. Estos momentos, que tienen sobre todo como propósito la transmisión de las ICP, son considerados como esenciales en el aseguramiento de la continuidad de los cuidados. Suponen la copresencia de los dos equipos: el que asume el servicio y el que lo deja. Durante estas reuniones, si bien el intercambio oral ha sido tradicionalmente importante, siempre se ha acompañado de los expedientes de los pacientes. Estos expedientes antes manuscritos, han sido reemplazados cada vez más por expedientes digitales, lo que clarifica las informaciones y mejora su categorización. Lo anterior redujo la importancia de la transmisión oral y en algunas ocasiones la redujo de manera significativa, incluso reemplazándola por una lectura individual de la información digital. Las directivas justifican lo anterior en nombre de la búsqueda de eficiencia, pues la larga duración de las reuniones moviliza o bloquea un número considerable de personas. Su reducción permitiría optimizar la distribución de recursos de personal.

Estos cambios - paso de la escritura en papel al expediente digital y reducción de la transmisión oral - implican diferentes vivencias. En lo que concierne a la escritura electrónica, las preguntas más frecuentes se relacionan con la manera de escribir las ICP, articulando síntesis y exhaustividad. A algunas enfermeras les preocupa escribir demasiado y a otras hacerlo muy poco. A menudo se preguntan en qué medida sus redacciones serán leídas y comprendidas por sus colegas: 'ser capaz de destilar la información es un trabajo muy difícil. ¿Qué hacer? Debemos transmitir lo que es importante y el medio utilizado influye (...) Si bien es útil (el computador personal), lo detesto: me fragmenta el pensamiento, estoy obligada a esquematizarlo todo'.

La lectura también inquieta: "Pensar que puedo leer un expediente sobre el estado del paciente en poco tiempo no es realista. Tengo que leer y concentrarme durante la lectura de manera a realmente entender quién es ese paciente'. A menudo las dudas se refieren al lugar, 'dónde insertar' los datos. Si bien las jóvenes resultan más competentes para este tipo de tareas, algunas de ellas consideran que las formas de categorización propuestas carecen de lógica.

A pesar de que para algunas el momento consagrado a la lectura personal del expediente corresponde a un momento gradual y personalizado de comprensión de la situación de los pacientes, la disminución del cambio de turno oral se vive en general como una pérdida en la transmisión de la información. La preocupación tiene que ver con la reducción de intercambios entre colegas así como con los riesgos de que muchas 
de las informaciones puedan perderse y de no poder consignar en el expediente digital del paciente los elementos de carácter vago, lo propio al 'ojo clínico' de la enfermera. ' Ese sexto sentido que hace que una enfermera diga 'no veo muy bien a ese paciente: ¿le prestarías atención?' Ahora bien, ¿cómo clasifica usted esto si está obligado sólo a escribirlo?' La consecuencia es que en ciertos servicios, aún si se da irregularmente, la manera de consignar la información se ha convertido en objeto de debates informales o formales, especialmente al momento de la llegada de nuevos colegas.

\subsubsection{Un cuestionamiento respecto a la imagen y al futuro de la profesión de enfermería}

Los OTD y las ICP son objetos manipulables y apropiables e 'invaden' los espacios de cuidado transformando no solo las prácticas sino también la imagen de la profesión de enfermería. En primer lugar, generan cuestionamientos relativos a su aceptación pues, de admitirse su utilidad, su uso representa para las enfermeras una fuente de opiniones encontradas que dependen solo parcialmente de la edad de las personas implicadas. Para algunas, se trata de elementos que entorpecen la relación con el paciente, al operar como pantallas que dificultan el encuentro y la escucha mutuos. Además, el uso y la aceptación de estas herramientas están condicionados a la eficacidad del sistema informático: en caso de sobrecarga del servidor o cuando sobrevienen problemas técnicos de un dispositivo particular, se manifiesta la sensación de perder el tiempo 'con esas máquinas'.

39 Así mismo, esos OTD suponen unas competencias específicas con las que las jóvenes tienen una mayor familiaridad. Frente a estas exigencias, las personas menos experimentadas muestran diferentes comportamientos: compromiso constante y valiente para aprender; doble redacción (en primer lugar manuscrita) de ciertos datos; solicitud de apoyo a las más experimentadas; o, en ciertos casos, modalidades de evitación de las tareas relacionadas con la redacción de los expedientes (redacciones reducidas, delegación a otras colegas). De otra parte, existe una comunidad de aprendizaje omnipresente relativa al uso de estos dispositivos, y caracterizada por una multiplicidad de modalidades de ayuda mutua.

Estos OTD cuestionan sobretodo la imagen profesional de las enfermeras: teclear $\mathrm{u}$ observar una pantalla son actividades que para algunas de ellas no corresponden a la imagen que tienen de sí mismas en tanto enfermeras, pues su propia actividad está concebida en referencia directa al paciente y/o en 'movimiento' en el servicio. Así, el personal no se siente cómodo 'viéndose' frente a un computador, pues tiene la impresión de actuar en otro contexto profesional (secretariado por ejemplo). A lo anterior se suman las preguntas de los pacientes acerca de las razones por las cuales las enfermeras pasan tanto tiempo frente al computador en lugar de 'hacer su trabajo'.

41 Además, estas herramientas cuestionan las modalidades de intercambio de experiencia entre colegas. Si bien las senior detentan una experiencia clínica importante, las jóvenes demuestran su experiencia en el uso de los OTD, lo que puede producir intercambios enriquecedores. Pero a veces sucede que las senior perciben la competencia para gestionar los OTD como de mayor importancia que la experiencia clínica. Esto genera en algunas de ellas un sentimiento de inutilidad de su propia experiencia y podría ocultar en las jóvenes su verdadera necesidad de aprovechar la experiencia clínica de sus colegas más experimentadas. Es posible observar a veces la 
confrontación de los dos grupos generacionales, que se manifiesta a través de la limitación de intercambios informales entre ellos, o a través de la afirmación de "no querer convertirse en...' (aquellas de la otra generación).

Así, los OTD para las ICP participan del proceso general y continuo de innovación de la enfermería e igualmente cuestionan su futuro. Le corresponden igualmente a este proceso las innovaciones locales, que dan fe de la creatividad y del pragmatismo del personal de enfermería. Por ejemplo, tal y como lo sugieren algunas profesionales, los riesgos relativos a las trabas en la relación con los pacientes, pueden resolverse adoptando diferentes estrategias de utilización de los OTD y/o comunicándose de otra manera con los pacientes: 'Por tanto, cuando estoy en la habitación y debo usar el laptop, le explico al paciente que no puedo hacer dos cosas al mismo tiempo y que debo concentrarme. Porque es también un asunto de seguridad (...). Entonces lo digo muy abiertamente: 'deme el tiempo de preparar su terapia (siguiendo las instrucciones en la pantalla), debo concentrarme y luego soy toda oídos".

Del mismo modo, entre las personas entrevistadas se evidencian dudas e imprecisiones respecto de la evolución de los cuidados de enfermería en los siguientes diez o quince años. Esto nos lleva a considerar este colectivo de profesionales como una 'comunidad profesional en transición' hacia un futuro que se percibe como incierto, en el marco de una tensión entre visiones optimistas (más rapidez en los procesos de cuidado, transparencia y trazabilidad, más seguridad, reducción de costos) y un pesimismo tecnocrático (el temor de que la 'auténtica' actividad relacional en la enfermería sea asfixiada por aspectos burocráticos y tecnológicos).

\subsection{Propuestas de intervención}

El conjunto de los resultados de esta investigación fue sometido a la validación de una parte de los profesionales implicados así como a la de otros investigadores y profesionales de los cuidados de enfermería, que confirmaron la importancia de los elementos aquí indicados y la necesidad de proponer intervenciones para abordar las problemáticas aquí evocadas. Dos propuestas se encuentran actualmente en elaboración: a) un estudio sobre las dinámicas de significación emergentes durante el proceso de transmisión de las ICP, teniendo en cuenta el conjunto de modalidades de comunicación presentes y proponiendo la elaboración de prototipos multimedia que ilustren la elección de situaciones típicas identificadas (Flandin et al., 2017; Ria, 2009); b) la promoción de un enfoque prospectivo, referente a los estudios sobre los sistemas de anticipación y las prácticas educativas en este terreno, con el fin de apoyar a los actores implicados en situaciones de cambio, de manera que puedan concebir de manera proactiva y participativa su propio porvenir. Lo anterior con el fin de facilitar sus procesos de anticipación y resiliencia y la elaboración interprofesional e intergeneracional de escenarios futuros (Godet et al., 2011; Poizat et al., 2016; Poli, 2017). 


\section{Investigación/intervención b: los efectos de la introducción de un nuevo progicial sobre la actividad individual y colectiva de los agentes judiciales de embargo}

La presentación que sigue trata de una intervención piloto que implicó un componente de análisis del trabajo y un componente de formación. Se realizó en dos etapas (2017-2018) con un grupo de agentes judiciales de embargo (AJE), a raíz de una solicitud hecha por el servicio de formación del Estado del cantón en cuestión y de la dirección general de ese servicio. La solicitud original se refería al deterioro global del estado de salud de los profesionales en el trabajo, así como a un retardo considerable en el procesamiento de los expedientes de los usuarios.

\subsection{Contexto y modalidad de realización de la investigación/ intervención}

Durante la primera etapa (2017) se realizaron un centenar de horas de observación del trabajo de los AJE, asumidas por tres analistas y repartidas a lo largo de una semana de inmersión, con el fin de comprender las especificidades de su actividad individual y colectiva y los elementos comunes y/o compartidos. Esta primera parte de la intervención puso en evidencia las características de la actividad de los AJE y reveló las dificultades provocadas por un progicial (SOFTPack2017), implementado en los diferentes servicios algunos meses antes y que estaba generando varias dificultades relativas, entre otros, a múltiples errores de codificación.

En la segunda etapa, llevada a cabo un año después, el equipo de analistas complementó el análisis del trabajo de los AJE con el fin de concebir procesos de formación. Esta vez, el análisis se enfocó en el trabajo de documentación del formulario que se utiliza, tanto en la ventanilla pública como en el domicilio, para constituir el expediente del deudor. Se realizaron unas cuarenta horas de observación y filmación de situaciones de trabajo de cinco AJE, así como entrevistas de autoconfrontación con los mencionados profesionales.

\subsection{Resultados}

En síntesis, esta base de información, enriquecida con los comentarios solicitados o espontáneos de los AJE, permitió: a) registrar, con el beneplácito de los profesionales implicados, huellas de este trabajo en forma de grabaciones de audio y de video; b) modelizar este trabajo con el fin de identificar sus rasgos típicos y/o críticos; c) escoger de la muestra observada los episodios que contenían esos rasgos típicos; d) concebir soportes para los procesos de formación, particularmente videoclips concebidos como artefactos con un potencial formativo; e) concebir y ejecutar dos sesiones de formación. Las líneas que siguen presentan los rasgos más importantes de la actividad de los AJE. Es importante aclarar que si bien la fase de documentación del citado formulario no constituye la totalidad de este trabajo, si constituye una parte central e ineludible. 


\subsubsection{El trabajo de los Agentes Judiciales de Embargo}

50 El servicio de embargos es el lugar al que se dirigen los acreedores cuyos deudores no quieren o no pueden saldar su deuda. Los AJE están organizados en equipos según sectores geográficos, compuestos por alrededor de cinco personas que trabajan colectivamente con el objetivo de cerrar cada expediente de la manera más eficiente posible. Uno de ellos funge como jefe de equipo, asumiendo la responsabilidad de los expedientes frente a los usuarios y a las autoridades.

51 La actividad de los AJE se compone de tres configuraciones principales diferenciadas pero articuladas, que permiten documentar cada expediente: a) la instrucción en solitario de los expedientes de los deudores en las oficinas virtuales de los AJE; b) la interacción en la ventanilla con los deudores que se presentan de mutuo propio o tras una citación; c) las visitas a los domicilios de los deudores. Completar la documentación de los expedientes resulta indispensable para hacer el inventario de los bienes embargables, y/o embargar dichos bienes o, en caso de ausencia de bienes, declarar la insolvencia.

52 La elaboración del expediente del usuario es el resultado de una co-construcción en la que el usuario es el protagonista principal. Aquí, la historia de las interacciones AJEusuario y el conocimiento por parte del AJE de la historia de los eventos relativos al expediente, juegan un rol central en el tratamiento fluido del expediente.

\subsubsection{La instrucción de los expedientes}

Cada AJE documenta el formulario en presencia del deudor, quien debe validarlo. Este proceso de documentación concierne simultáneamente un número elevado de expedientes que se encuentran en diferentes grados de progreso, todos de responsabilidad del AJE. El conjunto de expedientes evoluciona según la cantidad y la naturaleza de las operaciones realizadas por los profesionales. En cada sector, el trabajo individual y colectivo se inscribe fuertemente en este flujo temporal, que es simultáneamente el producto de este trabajo y la coerción que pesa sobre él. La documentación del formulario y el consecuente tratamiento de los expedientes se desarrollan siempre con ayuda del SOFTPack2017, el cual contiene informaciones registradas por los AJE relativas a las decisiones tomadas, a la documentación recogida durante su investigación en los diferentes momentos del proceso y al conjunto de piezas justificativas digitalizadas de cada expediente.

El análisis revela que se trata de una actividad extremadamente compleja, que va más allá de la simple documentación de rúbricas, y que es muchas veces llevada a cabo en una temporalidad larga, pues los deudores son a menudo usuarios habituales. En la ventanilla, el AJE utiliza herramientas e informaciones digitalizadas accesibles en dos pantallas, que le permiten registrar las informaciones recolectadas y simultáneamente verificar la veracidad de algunas de ellas y consultar el conjunto de los documentos justificativos.

\subsubsection{La entrevista en la ventanilla y durante las visitas a domicilio}

55 Mucho más que un simple procedimiento, el desarrollo de la entrevista llevada a cabo por el AJE para documentar el formulario, sirve para configurar una situación administrativa y jurídica que permite tomar una decisión acerca del posible embargo, 
de su monto, o de la declaración de insolvencia. El análisis evidencia que esta parte del trabajo, muy reglamentada por la ley, contiene aspectos éticos y compromete la responsabilidad de los AJE frente a cada uno de los expedientes, en los que tanto la situación del deudor como la del acreedor deben tomarse en consideración. Según los expedientes, dos exigencias potencialmente contradictorias estructuran en parte esta entrevista.

a - Entre confianza relativa y duda mesurada. Los AJE hacen gala de una gran habilidad para, sin perder de vista la precariedad de ciertas situaciones sociales de carácter dramático, frustrar las tentativas de enmascaramiento de los deudores. La sutilidad en la conducción de las entrevistas sirve para confundir a los usuarios que mienten o para poner a prueba y asegurar la buena fe del deudor. Esto genera una suerte de estado oscilante constante entre un principio de desconfianza y la decisión de confiar. Exige también una rápida y sostenida evaluación de la sinceridad o no del deudor a lo largo de toda la entrevista, y supone la necesidad de estrategias de cuestionamiento que buscan tranquilizar, dar confianza, o por el contrario, presionar con el fin de obtener una información de parte de un usuario que busca ocultar su verdadera situación financiera. Tal cosa requiere una gran vigilancia y una capacidad para apaciguar situaciones tensas, una resistencia frente a las tentativas de neutralización y tratos de ciertos deudores, así como una empatía controlada frente a ciertas situaciones difíciles.

Estos procesos de desenmascaramiento sutiles y siempre inciertos, requieren de parte de los AJE un conocimiento agudo de los expedientes de los deudores. Este conocimiento es posible gracias a la búsqueda de informaciones adicionales que surgen durante la entrevista (obtenidas a veces a través de un tercero durante las visitas a domicilio) y a la memorización de entrevistas anteriores. Ciertas informaciones se registran en el sistema informático, en documentos personales de los AJE y en su memoria.

b - Trabajar rápidamente. La obligación de eficacia y de búsqueda de rapidez en el tratamiento de los expedientes se relaciona con la necesidad de respetar los plazos reglamentarios y con serle fiel al ideal de eficacia en el servicio público. Sin embargo, la rapidez en el tratamiento de los expedientes no debe comprometer la calidad. La misma se evidencia en el respecto de los textos y en la preocupación de justicia y equidad frente a acreedores y deudores. Este dilema entre rapidez y calidad marca por consiguiente el flujo del trabajo individual y colectivo, desde la llegada a un sector del expediente y su tratamiento por parte de los asistentes, hasta su cierre por parte del AJE. No hay ni regularidad ni continuidad en el tratamiento, debido fundamentalmente al número de expedientes que deben gestionarse en paralelo; a los plazos legales de ciertas fases que imponen esperas; y a la diversidad de los comportamientos de los deudores. Por lo demás, la coerción de tiempo es también inmediata, debido a la presencia de otros deudores que esperan en el pasillo.

\subsubsection{Las implicaciones del SOFTPack2017}

Actualmente el SOFTPack2017 estructura y realiza una parte del trabajo individual y colectivo de los profesionales del servicio de embargos. Está concebido para la realización de algunas tareas sistemáticas tales como el envío de correos automáticos y para permitir una consulta transversal de la información de parte de los diferentes servicios implicados. Su implementación busca facilitar el trabajo de los profesionales y enmendar el retardo de los expedientes pendientes, en un contexto socio-económico en 
el que un porcentaje cada vez más importante de la población se encuentra en una situación de precariedad.

Este progicial transformó profundamente la actividad tal y como se desarrollaba antes de su implementación. Presentada a los colaboradores como portadora de simplificación del trabajo, la utilización del SOFTPack2017 revela otra cosa en la actividad cotidiana y aparece como generadora parcial de dificultades.

De acuerdo con las estimaciones de los colaboradores, el SOFTPack2017 es responsable de una ralentización del trabajo. La 'pérdida de tiempo' es importante: según cada caso, es necesario entre dos y cuatro veces más de tiempo para ejecutar tareas análogas a aquellas que se realizaban antes del SOFTPack2017 (los más expertos recuerdan los procedimientos anteriores que comparan con los actuales). Además de la acumulación de los expedientes en espera de tratamiento, existen otras consecuencias negativas asociadas a la actividad con el SOFTPack2017. Por ejemplo, se presentan errores de codificación recurrentes e incomprensibles; lentitud en el tratamiento entre las diferentes manipulaciones; bloqueos imprevisibles y aleatorios; dificultad en el acceso a la información útil durante ciertas fases del tratamiento de los expedientes; solicitudes recurrentes y acumulación de nuevos desarrollos informáticos difícilmente perceptibles; desaparición de los puntos de referencia en el avance del trabajo.

\subsubsection{Secuenciación del trabajo y estructuración a través de la tecnología digital}

El SOFTPack2017 modifica profundamente la actividad de los AJE, quienes parecen habérselo apropiado o se han habituado a fuerza de utilizarlo (la queja desapareció durante la segunda fase). Su utilización genera un cierto número de consecuencias que este grupo profesional comparte.

En la ventanilla, la documentación del formulario exige poner en relación y manipular múltiples veces un gran número de informaciones registradas en diferentes archivos. Este proceso, durante el cual el profesional debe simultáneamente prestar especial atención a los intercambios con el deudor, se realiza a través de la consulta de dos pantallas.

64 Antes de la implementación del SOFTPack2017, los AJE utilizaban un soporte en papel que les permitía hacer rápidamente una consulta transversal, desplegando en forma panorámica los archivos de manera que el tratamiento del expediente era cómodo y eficaz. La versión desmaterializada del trabajo de documentación ya no lo permite, pues el SOFTPack2017 ofrece una presentación secuenciada de los diferentes archivos limitada a las dos pantallas.

El trabajo de selección, cruce y síntesis de información resulta más largo. La consulta de los documentos, uno detrás de otro, depende de la memoria de los AJE y supone olvidos que requieren dar marchas atrás. Implica cansancio, ralentiza el proceso de tratamiento y genera dolores de cabeza ligados a los movimientos laterales de los ojos y a los esfuerzos de concentración. Para compensar estos inconvenientes y facilitar su trabajo, los AJE siguen imprimiendo los archivos para garantizar una confiabilidad en el tratamiento de los expedientes. Además, la versión papel de los expedientes permitía antes una clasificación ordenada en armarios, organizados de tal manera que con un solo vistazo era posible identificar los expedientes en espera, los expedientes procesados, y hacer una estimación rápida del tiempo necesario de trabajo, el todo consultando en diagonal los expedientes a tratar. Esto permitía una organización 
rápida del trabajo así como su planificación. De cierta manera, las clasificaciones materializaban la agenda de trabajo.

Puede constatarse entonces un fenómeno de volcamiento y ruptura de un trabajo que anteriormente se basaba en un principio de simultaneidad y de apreciación rápida, 'al alcance de la mano' de los AJE, hacia un trabajo linear y secuenciado con un progicial que constriñe y modifica la particular actividad de estos profesionales, tal y como la misma se desplegaba antes de su implementación.

\subsubsection{Fragilización de la posición profesional y desvalorización / proletarización de los saberes}

El análisis precedente indica que SOFTPack2017 afecta la cultura de acción de los profesionales. Entendemos por cultura de acción una serie de elementos: conocimientos, hábitos, valores, gustos, habilidades... que alimentan el trabajo cotidiano. Estos componentes son a la vez individuales y colectivos. A nivel individual, constituyen la fuente de la pericia, del compromiso y de las prestaciones de cada colaborador, en parte 'propia' a cada uno de ellos; a nivel colectivo, son en parte compartidos y cimientan la actividad colectiva de cada sector determinando sus especificidades. Esta cultura de acción fue puesta a prueba por el SOFTPack2017 y por un funcionamiento que privilegia la dimensión prescriptiva del trabajo. El desánimo generado por la multiplicidad de manipulaciones innecesarias y por los errores del SOFTPack2017 ligados a su inestabilidad, producen o tienen el riesgo de producir procesos de desentendimiento y de despersonalización en la manera de procesar los expedientes. Los AJE insisten en su sentido de responsabilidad personal que se ve comprometida en las decisiones que toman respecto de cada expediente, fundada en un conocimiento agudo de los expedientes, en la construcción a lo largo de las entrevistas con el deudor de una historia, en su percepción que describen como 'olfato' o intuición que los orienta en su cuestionamiento y en la toma de decisión. La obtención de informaciones proveniente de la fluidez de las investigaciones y de la imprevisibilidad de las interacciones sociales no se integra bien en este sistema digital impersonal. Además, los constantes errores generan una gran desconfianza hacia el SOFTPack2017, que exige un aumento en la vigilancia de parte de los AJE y un sentimiento de hacer el ridículo frente a ciertos deudores en los casos en los que se imputa un error al servicio.

68 A lo anterior se suma un sentimiento de desposesión que se puede traducir en el miedo a un futuro no muy lejano en el que se produzca un fenómeno de acaparamiento por parte de algoritmos de los componentes complejos, 'inteligentes', automatizando el trabajo de tratamiento de la información y la toma de decisiones situadas. El riesgo consiste en confinar el trabajo de los AJE a tareas de documentación de rúbricas predefinidas, relegando su trabajo a lo que Stiegler (2015a) denomina una 'proletarización de los saberes'.

69 En este contexto en el que los riesgos psicosociales están presentes, se perfila una pérdida del sentido del trabajo, así como la eventual pérdida de habilidades profesionales y un interrogante en la calidad en el tratamiento de los expedientes.

\subsection{La intervención formativa propuesta a los AJE}

La intervención tuvo como propósito visibilizar las habilidades desplegadas por los AJE durante la documentación del formulario, así como el debate acerca de las mismas. No 
se trataba por tanto de un complemento de formación para el uso del SOFTPack2017, sino más bien de un conocimiento del trabajo real atacado por esta versión digital y por las lógicas gerenciales asociadas. El objetivo era, a través del uso de seis videoclips de corta duración (entre tres y siete minutos), realizados por los analistas a partir de la edición de extractos escogidos de tomas de situaciones de trabajo y de sesiones de autoconfrontación, permitir a los AJE participantes durante la formación, describir las formas en las que estos abordan esos momentos de actividad, significar los valores que movilizan y la organización constantemente renovada que adoptan. La edición realizada en forma de narrativas, buscaba desencadenar perturbaciones en el acoplamiento actor-entorno de los participantes a la formación, a través de una implicación mimética frente a una experiencia similar de la suya propia. La proyección de estos videos se concibió para a) desencadenar debates colectivos; b) generar una posibilidad de inestabilidad en la manera de significar las situaciones de trabajo; c) provocar dinámicas de apropiación de los componentes típicos del trabajo; y d) producir movimientos de redistribución colectiva, a fin de fomentar la constitución de una comunidad de práctica capaz de mantener un trabajo cargado de significaciones para los profesionales en un contexto de transformación profunda (Durand, 2009, Durand et al, aceptado).

\section{Conclusion}

71 Al igual que muchos otros oficios, las profesiones de servicio están atravesadas por cambios organizacionales que le apuntan a la racionalización de la actividad, a su vez también caracterizada por procesos de estandarización, proceduralización, protocolización, etc. (Poizat et al., 2017). En el marco de estas mutaciones, la digitalización y especialmente el recurso masivo a los OTD para la gestión de información, ocupan un lugar importante gracias a sus promesas de mejoramiento de la calidad y de la productividad. (Djellal et al., 2007). En este sentido, los OTD son a la vez indicadores y actores de las transformaciones de la actividad de los profesionales de las profesiones de servicio. Son funcionales de tendencias ya en curso en el sector, a menudo reforzando la proceduralización de las prácticas, bajo el pretexto de control de calidad y la búsqueda de ganancias en productividad. Su introducción trae consigo transformaciones profundas del trabajo, que aquí discutimos según tres ejes: a) la relación entre la difusión de los OTD para la recopilación y tratamiento de la información y la evolución organizacional de las profesiones de servicio; b) el hecho de que el supuesto proceso de 'desmaterialización', entendido como el paso de expedientes en papel a expedientes digitales, implica transformaciones tanto técnicas, organizacionales y a nivel de las relaciones interprofesionales como de las modalidades de redacción y lectura de la documentación exigida, según la forma y el tipo de soporte utilizado; c) la necesidad de elaborar propuestas de formación que no le apunten en primer lugar al aprendizaje del uso del nuevo progicial (o al desarrollo de competencias digitales), sino que más bien consistan en intervenciones que permitan a los profesionales debatir colectivamente acerca de su trabajo real e inventar respuestas que propongan transformaciones en la actividad y que creen situaciones de apropiación individuales y colectivas duraderas.

Los profesionales de servicio participan de una multiplicidad de interacciones heterogéneas: con los usuarios, con los otros profesionales que ejercen el mismo oficio 
u otros, y con las herramientas informáticas (Motté et al., 2010). Su actividad se caracteriza por diferentes exigencias e intencionalidades que los conducen a veces, tal y como lo evidencian las dos investigaciones, a situaciones de 'doble presión', para responder a la vez tanto a las exigencias y preocupaciones de la empresa (a través de aquellas de los gerentes) como a aquellas de los usuarios. Las dos investigaciones muestran igualmente un aumento en la carga de trabajo, en parte debido a las condiciones de miseria social generalizada a su vez en aumento. Las nuevas tecnologías, usualmente pensadas como una solución a los problemas existentes, pueden convertirse gracias a la tecnología digital y a los algoritmos en una fuente de problemas, de alienación, de proletarización y de desindividuación de los profesionales de estos oficios, tendiendo a situaciones de 'sofocación' del trabajo (Poizat et al., 2017; Stiegler, 2015a). Más específicamente, la actividad de recopilación y tratamiento de información digital puede estar a la base de una proletarización de saberes necesarios, y sobre todo de una posible mutación del foco de estas profesiones hasta la fecha centradas en una relación de servicio público a los usuarios. Esto requiere otros saberes profesionales, otras comprensiones de lo que es la práctica profesional, así como esfuerzos relacionados con la articulación de las actividades individuales tanto con el colectivo profesional como con el colectivo transverso (constituido por la articulación entre profesionales de diferentes oficios), todos implicados en la ejecución del servicio requerido (Motté et al., 2010). Además, estos OTD llamados a facilitar la interacción, permiten al mismo tiempo controlar la actividad de los profesionales. Lo anterior es particularmente evidente en los dos casos presentados, en los que los progiciales aseguran un doble monitoreo de la situación de los pacientes/usuarios y del trabajo realizado por los profesionales. Estas herramientas digitales son entonces eficaces en la gestión de los servicios, de recursos humanos y de las finanzas. Las interacciones de los profesionales de servicio con los usuarios están atravesadas por los medios técnicos de los cuales disponen: por una parte, los OTD pueden facilitar el curso de las interacciones y su resultado; por la otra, los profesionales están llamados a dominar tanto su oficio como esos OTD, sin los cuales su trabajo es imposible.

El proceso de cambio de los expedientes en papel a los expedientes digitales, llamado a veces 'desmaterialización', implica simultáneamente transformaciones técnicas, organizacionales y de las relaciones interprofesionales. Este cambio no se reduce a un simple cambio de soporte, sino que requiere e induce una reorganización del sistema de trabajo y obliga a un cambio de mentalidad (Desaubry, 2009). Además, como lo subrayan Boucheix y Coiron (2008), es necesario tener en cuenta las implicaciones del paso de la información oral a la redacción de las comunicaciones por escrito y los diferentes niveles de estructuración de la anotación escrita exigida (Bachimont, 2001). Respecto a lo anterior, subrayamos la falsa evidencia de la noción de desmaterialización: los cambios de soporte simplemente conducen a un nuevo 'modo de materialización' y conllevan tanta técnica como el anterior (Leroi-Gourhan, 1964/1971; Pascal, 2004). Esto engendra nuevos cuestionamientos relativos a la dimensión técnica de la actividad humana e invita asimismo a una 'rematerialización' de los temas educativos en todos los ámbitos sociales (Poizat et al., 2017).

Retomamos tres de estos temas, puestos en evidencia por las dos investigaciones: a) una creciente formalización de la información que debe incluirse en los expedientes electrónicos, que debe apuntarle a las 'evidencias' y no a los elementos imprecisos, tales como las impresiones, intuiciones o ideas acerca del progreso del paciente o de la toma de decisión acerca de un usuario. Este aspecto es susceptible de cuestionar e incluso 
reducir las habilidades profesionales, a menudo basadas en la lectura implícita de un conjunto de elementos no siempre formalizables o preestablecidos; $b$ ) una discrepancia entre el sistema de tipificación de aquellos que elaboran la estructura de los expedientes electrónicos y de aquellos que los utilizan. Los individuos piensan y categorizan el mundo de maneras diferentes, que no son ni arbitrarias ni comparables a los mecanismos lógico-formales, pero que están basados en su experiencia sensorial, afectiva, social y cultural (Rosch, 1978; Violi, 2001). En este sentido, un desconocimiento de las particularidades del oficio puede dejar de lado aspectos esenciales que lo caracterizan, y los OTD podrían promover un neo-taylorismo en la medida en que su diseño no tenga en cuenta estos aspectos; c) finalmente, los expedientes electrónicos no parecen poder 'sustituir' completamente las otras formas y soportes de comunicación, sino que interactúan con ellas, complejizando el conjunto de la red socio-técnica de gestión de recopilación y tratamiento de la información.

75 La llegada de los OTD genera preguntas interesantes para los formadores, que exigen una reflexión profunda relativa a las competencias útiles en la sociedad actual. En efecto, la intervención de los formadores no puede circunscribirse a propuestas de formación que le apunten en primer lugar al aprendizaje del uso de la nueva herramienta digital. Se trataría más bien de intervenciones, en el marco de una lógica de desarrollo, que promuevan un debate colectivo de los profesionales acerca de su trabajo real individual y colectivo, con el fin de inventar respuestas para nuevos usos individuales y colaborativos eficaces, y así facilitar el proceso de apropiación / individuación.

76 Además, las diversas dificultades a las que se enfrentan los profesionales, subrayan la necesidad de concebir los OTD a partir de una comprensión aguda hecha con antelación de la actividad de los profesionales. La herramienta técnica debería suplir, sostener, aligerar y hacer posibles nuevos desarrollos de la actividad. No se trata entonces de adaptar el humano a los OTD, sino más bien de crear objetos técnicos que constituyan una ayuda para los humanos. Se trata en cierto sentido de remediar los efectos negativos, producidos por las presiones en el trabajo generadas por la ignorancia del consultor informático del trabajo real, y por las lógicas gerenciales que prescriben el trabajo sin tener en cuenta las exigencias y las necesidades concretas.

Las preguntas para el formador de adultos no conciernen únicamente la identificación de las competencias necesarias para esas nuevas disposiciones ('humano - no humano') en el lugar de trabajo, sino también en qué medida tener en cuenta el lugar de los OTD en el colectivo transverso (Motté et al., 2010), proponiendo no solamente una discusión colectiva acerca de la concepción de los OTD utilizados, sino también una reflexión sobre la actividad real de cada profesional implicado. Esto supone igualmente que el formador de adultos tenga de manera anticipada un lugar en los procesos de concepción (en colaboración con los otros creadores) de ambientes capacitantes, favoreciendo una reflexividad aumentada de los profesionales que actúan en situaciones cada vez más digitalizadas. 


\section{BIBLIOGRAFÍA}

Australian Commission on Safety and Quality in Health Care (2010). The OSSIE Guide to Clinical Handover Improvement. Sydney: ACSQHC.

Bachimont, B. (2001). Dossier patient et lecture hypertextuelle. Problématique et discussion. Les Cahiers du numérique, 2, 105-123.

Benghozi, P. J. (2011). Economie numérique et industries de contenu : un nouveau paradigme pour les réseaux. Hermès, 59, 31-37.

Benghozi, P. J., Gille, L., \& Vallée, A. (2009). Innovation and regulation in the digital age: A call for new perspectives. In P. Curwen, J. Haucap, \& B. Preissl (Eds.), Telecommunication markets drivers and impediments (pp. 503-550). Heidelberg: Physica-Verlag.

Boucheix, J.-M., \& Coiron, M. (2008). Analyse de l'activité de transmission écrite au cours des relèves de poste à l'hôpital. Activités, 1(5), 79-102.

Bourgeois E., \& Durand, M. (Eds.) (2012). Apprendre au travail. Paris: PUF.

Caroly, S., \& Weill-Fassina, A. (2007). En quoi différentes approches de l'activité collective des relations de services interrogent la pluralité des modèles de l'activité en ergonomie ? Activités, 4(1), 85-98. URL : http://activites.revues.org/1414 ; DOI: 10.4000/activites.1414.

De Montmollin, M. (1986). L'ergonomie. Paris: La Découverte.

Desaubry, L. (2009). La dématérialisation des dossiers documentaires : les enjeux et les techniques: étude de faisabilité réalisée pour le centre de documentation des Arts Décoratifs. Mémoire pour l'obtention du titre de «Chef de projet en ingénierie documentaire», Institut national des techniques de la documentation, Paris: CNAM.

Djellal F., \& Gallouj, F., (2007). La relation innovation-emploi dans les services: un bilan et un agenda de recherche. In M-C. Monnoyer \& P. Ternaux (Eds), Mondialisation des services, innovation et dynamiques territoriales (pp. 22-43). Paris: L'Harmattan.

Durand, M. (2014). Activité humaine, pratiques sociales et éducation des adultes. In J. Friedrich \& J. Pita (Eds), Un dialogue entre concepts et réalité en formation des adultes (p.15-39). Dijon: Raison et Passions.

Durand, M. (2009). Analyse du travail dans une visée de formation: Cadres théoriques, méthodes et conception. In J-M. Barbier, E. Bourgeois, G. Chapelle, \& J.C. Ruano-Borbalan (Eds.), Encyclopédie de la formation (pp. 827-856). Paris: PUF.

Durand, M., \& Filliettaz, L. (Eds) (2009). Travail et formation des adultes. Paris: PUF

Durand, M., Goudeaux, A., Poizat, G., \& Sarmiento Jaramillo, J. (sous presse). Des films pour analyser le travail et documenter des situations de formation. Images du Travail - Travail des images.

Falzon, P., \& Lapeyrière, S. (1998). L'usager et l'opérateur : ergonomie et relations de service. Le Travail Humain, 61, 69-90.

Flandin, S., Seferdjeli, L., Bailly, M.-C., Goudeaux, A., Durand, I., Schmidt, K., Schot, S. (2017). Fonder la conception d'un environnement de formation sur l'analyse du travail: le cas de techniciens en radiologie médicale. 52ème Congrès International Société d'Ergonomie de Langue Française. Toulouse, France. 
Geslin, Ph. (1999). L'apprentissage des mondes. Une anthropologie appliquée aux transferts de technologies. Paris: Maison des sciences de l'homme.

Geslin, Ph. (2002). Les formes sociales d'appropriations des objets techniques, ou le paradigme anthropotechnologique. Ethnographiques.org, 1. www.ethnographiques.org/2002/Geslin

Geslin, Ph. (2005). The development of anthropotechnology in the social and human sciences: its applications on fieldworks. In P. Carayon (Eds.), Human factors in organizational design and management (pp. 455-460). Santa Monica: IEA Press.

Geslin, Ph. (2006). Le politique et le scientifique dans la pratique anthropotechnologique. Travailler, 1(15), 149-163. https://doi.org/10.3917/trav.015.0149

Geslin, Ph. (2012). La circulación de los hombres y las técnicas. Reflexiones sobre la antropotecnología. Laboréal, 8, 31-40.

Havelange, V. (2005). De l'outil à la médiation constitutive : pour une réévaluation phénoménologique, biologique et anthropologique de la technique. Arob@se, 1, 8-45.

Godet, M., \& Durance, P. (2011). La prospective stratégique pour les entreprises et les territoires. Paris: Dunod.

Leroi-Gourhan, A. (1971). El gesto y la palabra (Trad. F. Carrera) Caracas: Publicaciones de la Universidad Central de Venezuela. (Original en francés, 1964)

Motté, F., \& Haradji, Y. (2010). Construire la relation de service en considérant l'activité humaine dans ses dimensions individuelles et collectives. In G. Valléry, M.-C. Le Port, \& M. Zouinar (Eds.), Ergonomie et conception de produit et de services médiatisés (pp. 11-35). Paris: PUF.

Pascal, R. (2004). Critique de la dématérialisation. Communication et langages, 140, Dossier : Du «document numérique» au «textuel», 55-68. https://doi.org/10.3406/colan.2004.3268; www.persee.fr/doc/colan_0336-1500_2004_num_140_1_3268

Peirce, C.S. (1994). The collected paper of Charles Sanders Peirce (Volumes I-VIII). Charlottesville, VA : Intelex.

Poli, R. (2017). Strategie di futuro in classe. Esperienze, metodi, esercizi. Trento: Edizioni Provincia Autonoma di Trento - IPRASE.

Poizat, G., \& Goudeaux, A. (2014). Appropriation et individuation: un nouveau modèle pour penser l'éducation et la formation? Raisons éducatives 14, 13-38.

Poizat, G., \& Durand, M. (2017). Réinventer le travail et la formation des adultes à l'ère du numérique: état des lieux critique et prospectif. In G. Poizat \& M. Bétrancourt (Eds.), Technologies numériques et formation des adultes: Enjeux et perspectives (pp. 19-44). Genève: Université de Genève. Poizat, G., Durand, M., \& Theureau, J. (2016). Challenges of activity analysis oriented towards professional training. Le Travail Humain, 79, 233-258.

Ria, L. (2009). De l'analyse de l'activité des enseignants débutants en milieu difficile à la conception de dispositifs de formation. In M. Durand \& L. Filliettaz (Eds.), La place du travail dans la formation des adultes (pp. 217-243). Paris: PUF.

Rosch, E. (1978). Principles of categorization. In E. Rosch \& B. B. Lloyd (Eds.), Cognition and categorization (pp. 27-48). Hillsdale: Erlbaum.

Salini, D. (2018). Pratica infermieristica e dispositivi digitali. Implicazioni della diffusione di dispositivi digitali per l'informazione e la comunicazione nella pratica infermieristica. Report di ricerca. Lugano: Istituto universitario federale per la formazione professionale. 
Sartre, J.P. (2004). El ser y la nada. (Trad. J. Valmar). Losada Editorial (Original en francés, 1943)

Schütz, A. (2010). Essais sur le monde ordinaire. Paris: Éditions du Félin.

Simondon, G. (2005). L'individuation à la lumière des notions de forme et d'information. Grenoble:

Million

Simondon, G. (2008). El modo de existencia de los objetos técnicos. (Trad. M. Martínez y P. Rodríguez). Buenos Aires: Prometeo Libros. (Original en francés, 1989).

Steiner, P. (2010). Philosophie, technologie et cognition : état des lieux et perspectives. Introduction au dossier. Intellectica, 53-54(1-2), 7-40.

Stiegler, B. (1993). Temps et individuation technique, psychique, et collective dans l'œuvre de Simondon. Futur antérieur, 19-20,

http://www.multitudes.net/temps-et-individuation-technique/

Stiegler, B. (2014). Digital Studies. Limoges: FYP.

Stiegler, B. (2015a). La société automatique: Tome 1. L'avenir du travail. Paris : Fayard.

Stiegler, B. (2015b). Lo que hace que la vida merezca ser vivida: de la farmacología (Trad. N. Cortés). Averigani. (Original en francés, 2010).

Theureau, J. (2006). Le cours d'action: méthode développée. Toulouse: Octarès.

Theureau, J. (2010). Les entretiens d'autoconfrontation et de remise en situation par les traces matérielles et le programme de recherche «cours d'action». Revue d'Anthropologie des Connaissances, 4(2), 287-322. https://doi.org/10.3917/rac.010.0287

Theureau, J. (2012). Antropología cognitiva y antropotecnología. Laboreal, 8, 47-54.

Theureau, J. (2015). L'enaction et l'expérience. Toulouse: Octarès.

Valléry, G. \& Leduc, S. (2005). Contribution ergonomique à l'analyse des relations de service exemple de professionnalisation d'une fonction d'accueil en bureau de poste. Le travail humain, 68(2), 153-189. DOI: 10.3917/th.682.0153.

Varela, F. J., Thompson, E., \& Rosch, E. (1992). De cuerpo presente (Trad. C. Gardini). Barcelona: Editorial Gedisa. (Original en inglés, 1991).

Violi P. (2001). Meaning and Experience. Bloomington: Indiana University Press.

Wisner, A. (1984/2012). A antropotecnologia, ferramenta ou engodo? Laboreal, 8, (2), 15-31 http:// laboreal.up.pt/revista/artigo.php?id=48u56oTV658223577:7434;6352 .

Wisner, A. (1997). Anthropotechnologie: vers un monde industriel polycentrique. Toulouse: Octarès.

\section{NOTAS}

1. Un embargo es un procedimiento por medio del cual un acreedor solicita la ejecución de una obligación, generalmente avaluada en dinero 


\section{RESÚMENES}

En las profesiones de servicio, la digitalización transforma la actividad de los profesionales y su relación con los usuarios. Esto se manifiesta particularmente en la gestión de la información de los usuarios. Esta contribución explora las implicaciones de la digitalización de la información (de pacientes y clientes) según dos estudios preliminares realizados en Suiza en el ámbito de la enfermería y de los agentes judiciales de embargo. Realizados según la perspectiva de la antropotecnología educativa, estos estudios muestran que la introducción de objetos técnicos digitales (OTD) conlleva profundas transformaciones en el trabajo. La contribución analiza: a) la relación entre la difusión de los OTD y la evolución organizacional de las profesiones de servicio, b) la inherente complejidad de las transformaciones de las modalidades de soporte y comunicación, y c) la necesidad de proponer procesos de formación que le apunten a la preparación o a la reparación de las prácticas digitalizadas.

No setor de serviços, a digitalização está a transformar a atividade individual e coletiva dos profissionais e sua relação com os utilizadores. Sem limitação, isto manifesta-se sobretudo na digitalização que diz respeito à gestão de informações dos utilizadores. Esta contribuição explora as implicações da digitalização das informações (clientes, pacientes), baseada em dois estudos preliminares realizados na Suíça, no âmbito das práticas de enfermaria e dos oficiais de justiça de uma Procuradoria. Realizados segundo a perspetiva da antropotecnologia educacional, estes estudos demonstram que a introdução de objetos técnicos digitais (OTD) leva a profundas transformações no trabalho, que são analisadas em três linhas: a) a relação entre a difusão das OTD e a evolução organizacional das profissões de serviços, b) a complexidade inerente às transformações das modalidades e suportes de comunicação, e c) a necessidade de propor processos de formação que visem a preparação ou a reparação das práticas digitais.

Dans les métiers de services, la digitalisation transforme l'activité individuelle et collective des professionnels ainsi que la relation aux usagers. Sans s'y limiter, ceci est particulièrement manifeste quand cette digitalisation concerne la gestion des informations sur les usagers. Cette contribution explore les implications de la numérisation des informations (clients, patients), sur la base de deux études liminaires menées en Suisse, dans le domaine des pratiques infirmières et des huissiers d'un office de poursuites. Conduites en référence à une perspective d'anthropotechnologie éducative ces études montrent que l'introduction d'objets techniques numériques (OTN) est porteuse de profondes transformations du travail, discutées selon trois axes : a) le rapport entre la diffusion des OTN et l'évolution organisationnelle des métiers de service, b) la complexité inhérente aux transformations des modalités et supports de communication, et c) la nécessité de proposer des interventions formatives visant soit la préparation soit la réparation des pratiques digitalisées.

In the services sector professions, digitalization is transforming the individual and collective activity and the relationship with the users. Though not exclusively, this aspect is particularly visible when digitalization refers to the management of the user's information. This contribution explores the implications of the information's digitalization (of clients, patients). It is based on two preliminary studies carried out in Switzerland, about the practice of nurses and bailiffs. The studies followed the view of educational anthropotechnology and showed that the introduction of technical digital objects (TDO) leads to profound transformations in work. These are discussed according to three lines of thought: a) the relationship between the wide circulation of the TDO and the organizational evolution of services sector professions, b) the inherent complexity in the 
transformation of communication's modalities and media, and c) the need to propose educational interventions addressing digital practices preparation or reparation.

\section{ÍNDICE}

Mots-clés: métiers de services, anthropo-technologie, objets techniques numériques, analyse de l'activité, organisation du travail

Palabras claves: profesiones de servicio, antropo-tecnología, objetos técnicos digitales, análisis de la actividad, organización del trabajo

Palavras-chave: profissões de serviços, antropotecnologia, objetos técnicos digitais, análise da atividade, organização do trabalho

Keywords: services sector professions, anthropotechnology, technical digital objects, activity analysis, work organization

\section{AUTORES}

\section{DELI SALIN}

Istituto universitario federale per la formazione professionale IUFFP, Dipartimento Ricerca e sviluppo, via Besso 84, CH 6900 Lugano

deli.salini@iuffp.swiss

\section{JUANA SARMIENTO JARAMILLO}

Université de Genève, Faculté de psychologie et des Sciences de l'éducation, bd du pont d'Arve 40, CH 1211 Genève, juana.sarmiento@etu.unige.ch / To.Be Consultores, Calle 136 \# 74-65, Bogotá, Colombia

juana.sarmiento@tobe.com.co

\section{ANNIE GOUDEAUX}

Université de Genève, Faculté de psychologie et des Sciences de l'éducation, bd du pont d'Arve 40, $\mathrm{CH} 1211$ Genève

annie.goudeaux@unige.ch

\section{GERMAIN POIZAT}

Université de Genève, Faculté de psychologie et des Sciences de l'éducation, bd du pont d'Arve 40, CH 1211 Genève

germain.poizat@unige.ch 